\section{¿Podemos confiar en el etiquetado nutricional de los alimentos en Chile?}

\author{
INÉS URQUIAGA ${ }^{\mathrm{a}}$, MAGDALENA LAMARCA ${ }^{\mathrm{b}}$, PAULINA JIMÉNEZc \\ GUADALUPE ECHEVERRÍA $^{\mathrm{d}}$, FEDERICO LEIGHTON ${ }^{\mathrm{e}}$
}

\section{Assessment of the reliability of food labeling in Chile}

Background: Nutrition labeling is a tool that helps people to make healthy choices when choosing foods and drinks for consumption. Easy to understand information promotes rational choices. However the implementation of this information by industry and their proper use by consumers is complex. A key aspect is the existence of appropriate laws and regulations. Fortunately, Chile has a mandatory guideline for labeling packed food products. Though, the correctness of the information appearing in the label is essential. Aim: To evaluate the veracity of nutritional information appearing on food labels in our country. Material and Methods: We tabulated the information on the labels of over 1,000 packed products and analyzed it using globally accepted tables as reference. We included cereals, vegetables, fruits and juices, nuts, legumes, dairy, fish and shellfish, meat, oils and fats and sweets. Results: Ninety percent of the information appearing in the labels of analyzed products was presumably correct. However, there were great variations among food groups. Conclusions: This information allowed evaluate the application of the Food Health Regulations reform implemented in 2006, specifically Article 115. The possible improvements to achieve the original aims of the initiative are discussed.

(Rev Med Chile 2014; 142: 775-781)

Key words: Dietary carbohidrates; Dietary fat; Dietary proteins; Food labeling; Government regulation.

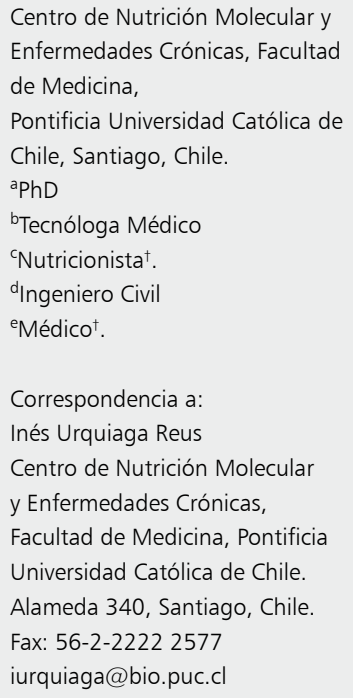

$\mathrm{E}$ l etiquetado nutricional es una herramienta que facilita a las personas tomar decisiones saludables al elegir alimentos y bebidas para su consumo habitual. Una información veraz y fácil de comprender podría promover elecciones más racionales, pero el proceso de implementación de la entrega de esta información por parte de la industria y su adecuado uso por parte de los consumidores son temas complejos, siendo clave la existencia de legislación y regulaciones que aseguren una adecuada aplicación de este tipo de iniciativas.

En Estados Unidos de Norteamérica, la Food and Drug Administration (FDA) ha hecho progresos importantes como parte de un esfuerzo constante por mejorar la información sobre la composición de alimentos entregada a los consumidores, con el fin de disminuir el riesgo de enfermedades crónicas ${ }^{1-8}$. Lo mismo ha ocurrido en la Comunidad Económica Europea, donde en enero de 2007 entró en vigencia una nueva regulación sobre Declaraciones de Nutrición y Salud en los Alimentos, actualizada en enero de 2013 ${ }^{9-12}$.

Nuestro país, cuyo perfil epidemiológico ha mostrado un incremento sostenido en la prevalencia de enfermedades crónicas no trasmisibles relacionadas con la alimentación ${ }^{13}$, también cuenta con una normativa de vigencia obligatoria para el rotulado de productos alimenticios envasados. Así, a fines del año 2006 entró en vigor el Decreto 
Supremo No 57 del 16 de febrero de 2005, que modificó al Decreto Supremo No 977/96, ambos del Ministerio de Salud, en relación al etiquetado de los alimentos, para ayudar a los consumidores a seleccionar una alimentación más saludable que contribuya a prevenir la obesidad y enfermedades relacionadas $^{14-15}$. El Reglamento Sanitario de los Alimentos (RSA) se reformó, incorporando la obligatoriedad de incluir el etiquetado nutricional que informa el contenido de nutrientes en los productos alimenticios que se comercializan envasados (Artículo 115 RSA).

Según el Artículo 115 del RSA, la información mínima que debe aparecer en la etiqueta de los productos alimenticios es el contenido en energía (expresado en kilocalorías $(\mathrm{kcal})$ ), proteínas, grasa total y carbohidratos disponibles (expresados todos en gramos); y sodio (expresado en miligramos). Se entiende por carbohidratos disponibles a todos los hidratos de carbono con exclusión de la fibra dietética. En los productos que contienen 3 o más gramos de grasa por porción de consumo habitual, debe informarse, además de la grasa total, el contenido de colesterol y de ácidos grasos saturados, trans, mono insaturados y poli insaturados. Según la Ley, los valores que figuran en la declaración de nutrientes, pueden ser valores medios ponderados, provenientes del análisis químico del producto certificado o provenientes de tablas de referencia, debidamente reconocidas por organismos nacionales o internacionales que informen sobre ese producto.

Posteriormente, la consulta pública realizada entre octubre y diciembre de 2010 -consulta pública para optimización del Etiquetado Nutricional de Alimentos-, incorporó en forma obligatoria cambios importantes al RSA (Artículos 115 y 116), incluyendo la rotulación de la cantidad de azúcares totales y cualquier otro carbohidrato disponible (hidratos de carbonos endulzantes, mono y disacáridos refinados, concentrados y/o cristalizados) en vez de azúcar (sacarosa natural) y de fibra soluble e insoluble cuando exista una declaración de propiedades saludables respecto de la fibra dietética.

Para destacar las cualidades de un alimento o producto en cuanto a determinados nutrientes, sólo estaba permitido el uso de los siguientes descriptores: Libre, Bajo aporte, Buena fuente, Alto, Reducido, Liviano, Fortificado, Extra magro, Muy bajo en sodio. El Reglamento precisa que las palabras "Diet" y "Light" son nombres de fantasía que sólo sugieren una propiedad nutricional.

En el Diario Oficial se publicaron en octubre de 2009, las normas técnicas sobre directrices nutricionales para los mensajes que se utilicen para declarar propiedades saludables y funcionales de los alimentos, consistentes en la asociación entre un alimento, un nutriente $\mathrm{u}$ otra sustancia y una condición de salud. Se estableció que los mensajes saludables no se deben utilizar en alimentos que por porción de consumo habitual contienen cantidades superiores a 13,0 g de grasa total o 4,0 g de grasa saturada o $60 \mathrm{mg}$ de colesterol o 480 mg de sodio.

Además, se establece que los descriptores: libre, bajo aporte, reducido y liviano en colesterol sólo podrán aplicarse a alimentos que sean libres de grasa trans (máximo 0,2 g de ácidos grasos trans por porción de consumo habitual) y que contengan por porción de consumo habitual máximo 2 g de grasa saturada.

Luego, el 2 de enero de 2011 se publicó en el diario oficial modificaciones al RSA, que permiten el uso de palabras en otro idioma o palabras de fantasía, que se asocien inequívocamente con características nutricionales, tales como "light", "diet", "high", "lite", "low", "delgadissimo", "flakin" y "soft", entre otras, siempre que cumplan y rotulen con los parámetros de alguno de los descriptores autorizados en el RSA.

El 6 de julio de 2012, se promulgó la Ley sobre Composición Nutricional de los Alimentos y su Publicidad -Ley 20.606-, que obliga a rotular los alimentos que contengan elevados contenidos de energía, grasas saturadas, grasas trans, azúcares y sal, como "Alto en...".

Por otra parte, en el rótulo de ingredientes debe figurar la lista de todos los ingredientes y aditivos que componen el producto, con sus nombres específicos, en orden decreciente de su contribución al peso o volumen final.

Cabe destacar que los alimentos fraccionados y/o envasados a solicitud del consumidor, tales como trozos de carne, queso, fiambre, fruta, pescado y otros, quedan excluidos de la obligatoriedad de informar la composición nutricional del producto. Sin embargo, si el establecimiento opta por indicarlos, deberá cumplir con las normas de etiquetado señaladas en el RSA.

Conscientes de la importancia del etiquetado nutricional para una política pro-activa de 
protección de la salud de las personas a través de una alimentación adecuada en Chile, nos interesó examinar la forma en que se está aplicando la entrega de información en el etiquetado de los alimentos. Lo primero es que las personas reciban una información correcta sobre la composición química del alimento en el momento de seleccionar un producto. Por esto, decidimos evaluar específicamente la verosimilitud de la información nutricional obligatoria rotulada en los alimentos disponibles en Chile. Este estudio analiza solamente la información nutricional obligatoria según el Art. 115 del RSA vigente, no considera los mensajes nutricionales saludables, ni de advertencia. Con este objeto, tabulamos la información que aparece en las etiquetas de más de 1.000 productos envasados, analizamos la congruencia existente entre calorías, composición de macronutrientes, ingredientes declarados y tipo de alimento, consultando Tablas de referencia mundialmente aceptadas ${ }^{16-18}$. Los productos estudiados corresponden a cereales, vegetales, frutas y jugos, frutos secos, legumbres, lácteos, pescados y mariscos, carnes, aceites y grasas y golosinas. Los resultados de este análisis contribuyen a establecer cómo se está aplicando el Art 115 del RSA vigente y advierten el modo de perfeccionar su aplicación para dar mayor impulso al objetivo original de esta iniciativa.

\section{Procedimiento y análisis}

La evaluación del etiquetado nutricional se realizó en un total de 1.020 productos, de los cuales 960 están obligados por la Ley a informar el contenido de nutrientes, mientras que otros 60 informan su composición nutricional en forma voluntaria, sin estar obligados a hacerlo. De la información mínima exigida por el Art. 115 del RSA vigente se analizó: el valor energético en Kcal, las cantidades de proteínas, carbohidratos disponibles y grasa total, y en los productos que contienen 3 o más gramos de grasa por porción de consumo habitual, el contenido de ácidos grasos saturados, mono insaturados, poli insaturados y ácidos grasos trans. La cantidad de cualquier otro nutriente exigida por una declaración de propiedades nutricionales no se consideró. La distribución de los productos estudiados fue la siguiente: cereales $7,7 \%$, vegetales $10,1 \%$, frutas y jugos con fruta $6,7 \%$, frutos secos $1,0 \%$, legumbres $1,7 \%$, lácteos $16,4 \%$, pescados y mariscos $4,4 \%$, carnes rojas, blancas y procesadas $16,6 \%$, aceites y grasas $12,6 \%$ y golosinas $22,8 \%$.

Se hizo un registro fotográfico de las etiquetas de los productos para su tabulación, el que se realizó mayoritariamente en un supermercado de la ciudad de Santiago, durante el mes de octubre del año 2007. Se seleccionaron al azar productos de consumo habitual, de distintas categoría de alimentos, en la selección no se utilizó ningún criterio estadístico, se eligieron productos de consumo masivo y de diferentes marcas. Los 960 productos representan $30 \%$ del total de productos envasados, obligados a rotular, en el mercado ${ }^{19}$.

El análisis de los datos obtenidos de las etiquetas, se hizo en base a la composición química del producto por cada 100 gramos (g) de éste. Para determinar la verosimilitud del etiquetado nutricional, se examinó:

1. La correspondencia de la energía según el contenido de proteínas $(4 \mathrm{kcal} / \mathrm{g})$, grasas $(9 \mathrm{kcal} / \mathrm{g})$ y carbohidratos $(4 \mathrm{kcal} / \mathrm{g})$.

2. La concordancia entre las grasas totales y el desglose de los diferentes tipos de grasas en los alimentos que indican los distintos tipos de este nutriente.

3. La correspondencia de la composición nutricional declarada en la etiqueta y los ingredientes del alimento procesado en cuestión.

4. La congruencia de la información declarada en la etiqueta con datos de Tablas de referencia del alimento en cuestión. Las Tablas de referencia utilizadas fueron la Composición Química de Alimentos Chilenos ${ }^{18}$, la Composición de los Alimentos de la USDA ${ }^{16}$ y la Composición de Alimentos Españoles, de la Universidad de Granada ${ }^{17}$.

Cada etiqueta nutricional se clasificó como correcta o incorrecta. Se consideró correcta cuando cumplía con las normas exigidas por el RSA y con los 4 puntos anteriores. Se consideró incorrecta cuando no cumplía con el RSA (por ejemplo: se informaban hidratos de carbono totales en lugar de carbohidratos disponibles o la información era incompleta) o con alguno de los 4 puntos anteriores. Las Tablas de referencia se utilizaron solamente como un apoyo, para decidir si la información nutricional podía o no corresponder al alimento en cuestión (por ejemplo: un aceite de oliva que 
informa $13 \%$ de grasa mono insaturada siendo que las Tablas de referencia informan entre 60 y 80\%). En este análisis se aceptó una variación de hasta $20 \%$ respecto a un alimento de referencia, similar a los límites de tolerancia que acepta el RSA para alimentos que no destacan mensajes nutricionales.

Los resultados se presentan en Tablas que muestran el número y la frecuencia de etiquetas incorrectas por grupo de alimentos (Tabla 1) y también se reporta una clasificación de los errores encontrados más frecuentemente en los distintos grupos (Tabla 2).

\section{Calidad de la información}

La Tabla 1 muestra la cantidad y proporción de información incorrecta en el etiquetado nutricional de los alimentos analizados, en los cuales el etiquetado nutricional es obligatorio de acuerdo al Art 115 del RSA vigente en nuestro país. En promedio, 9,6\% del etiquetado nutricional obligatorio presenta algún tipo de inexactitud o error.

El grupo de los vegetales tiene el mayor porcentaje (50\%) de información equivocada (Tabla 1). Los vegetales frescos muestran la mayor parte

Tabla 1. Cantidad y proporción de información incorrecta en el etiquetado nutricional de alimentos según grupos de alimentos analizados

\begin{tabular}{|lcccc|}
\hline Alimento & $\mathbf{n}$ & \% del total & Errores & \% errores \\
Cereales & 75 & 7,8 & 2 & 2,7 \\
Vegetales & 98 & 10,2 & 49 & 50,0 \\
Frutas y jugos & 59 & 6,1 & 2 & 3,4 \\
Frutos secos & 10 & 1,0 & 2 & 20 \\
Legumbres & 17 & 1,8 & 0 & 0 \\
Lácteos & 165 & 17,2 & 3 & 1,8 \\
Pescados-mariscos & 40 & 4,2 & 4 & 10 \\
Carnes & 134 & 14,0 & 3 & 2,2 \\
Grasas & 129 & 13,4 & 9 & 7,0 \\
Golosinas & 233 & 24,3 & 18 & 7,7 \\
Total & 960 & 100 & 92 & 9,6 \\
\hline
\end{tabular}

n: número de alimentos analizados. \% del Total: porcentaje del total de alimentos analizados. Errores: número de alimentos analizados con información incorrecta. \% Errores: porcentaje de información incorrecta en el grupo.Del etiquetado nutricional obligatorio, Art. 115 del RSA vigente, se analizó: el valor energético; las cantidades de proteínas, carbohidratos disponibles y grasa total, y en los productos que contienen 3 o más gramos de grasa por porción de consumo habitual, el contenido de ácidos grasos saturados, monoinsaturados, poliinsaturados y ácidos grasos trans. La cantidad de cualquier otro nutriente exigida por una declaración de propiedades nutricionales no se consideró.

Tabla 2. Tipo de errores más frecuentes encontrados en el etiquetado nutricional obligatorio

\begin{tabular}{|lcc|}
\hline Tipo de errores & n de errores & \% del total de errores \\
Información no corresponde con el alimento o con sus ingredientes & 27 & 29,3 \\
\hline Informan carbohidratos totales y no disponibles & 39 & 42,4 \\
Información incompleta & 13 & 14,1 \\
Información de grasas totales no tiene relación con grasas parciales & 7 & 7,6 \\
Información de calorías no tiene relación con composición nutricional & 6 & 100 \\
\hline Total & 92 & 6,5 \\
\hline
\end{tabular}

n de Errores: número de errores. \% del total de Errores: porcentaje del total de errores. Ver glosa Tabla 1. 
de los errores. Del análisis del tipo de errores más frecuentes, este grupo muestra que la etiqueta informa el contenido de hidratos de carbono totales, el que comprobamos como copiado de Tablas de referencia, sin indicar los hidratos de carbono disponibles que exige el RSA. En varios otros casos se pudo constatar que las empresas reportan la información de las Tablas de referencia en forma errada, como informar la composición del producto seco (romero, estragón, jengibre, entre otros) siendo que el producto es fresco.

Los pescados y mariscos y los frutos secos muestran 10 y $20 \%$ de información errónea, respectivamente. En el caso de pescados y mariscos, la mayoría de los errores se encuentra en los pescados congelados. En ambos casos, las incorrecciones se encuentran principalmente asociadas a la información del contenido de grasa total y al tipo de grasas que da cuenta de la grasa total.

Las categorías de alimentos con menos información incorrecta son: frutas y jugos de fruta, cereales, carnes, lácteos y legumbres, presentando 3,$4 ; 2,7 ; 2,2 ; 1,8 ; \mathrm{y} 0 \%$, respectivamente. En frutas y jugos, las equivocaciones ocurren exclusivamente para la fruta fresca y deshidratada mientras que en los cereales se presentan en harinas, como harina de maíz. En ambos grupos, la inexactitud de la información radica en el reporte de carbohidratos totales versus disponibles. En el grupo de las carnes, los errores se encuentran en carnes procesadas $\mathrm{y}$ estos se deben principalmente a un informe incorrecto del contenido calórico. En lácteos, el grupo de los quesos concentra las irregularidades, principalmente porque las grasas informadas no corresponden al contenido lipídico real de este tipo de alimentos.

El grupo de las grasas (aceites, mantecas, mantequillas, margarinas y aderezos grasos) presenta $7,0 \%$ de información dudosa, concentrándose los errores en aceites, mantequillas y aderezos. Estos se deben principalmente a información incompleta o a que la composición grasa no corresponde realmente al tipo de alimento.

El grupo de las golosinas es heterogéneo, presentando $7,7 \%$ de la información incorrecta. Los errores se presentan mayoritariamente en las golosinas dulces (galletas, "galletones", "brownies", alfajores, "cuchuflí", y otros) y en los jugos en polvo. Esto se debe básicamente a información incompleta o información que no concuerda con los ingredientes del producto.
En el análisis global del tipo de errores detectados en el etiquetado nutricional obligatorio, $42,4 \%$ se debe a que se informa el contenido de carbohidratos totales en lugar de los carbohidratos disponibles (Tabla 2). El 29,3\% pertenece a información de una composición nutricional que no corresponde al producto, tales como una galleta preparada con harina de trigo que no contiene proteína o un queso de leche de bovino que no contiene grasa saturada. En 14,1\%, la información es incompleta, como la falta del informe del tipo de grasa, siendo que el producto tiene más de 3 g de grasa por porción. En 7,6\% se informa un contenido de grasa total que no guarda relación con la suma de los distintos tipos de grasa presente, como el caso de la información de un pescado que reporta $17 \mathrm{~g}$ de grasa total y 5, 2,1 y 2,5 g de grasa saturada, mono insaturada y poli insaturada, respectivamente. En 6,5\% se informa una cantidad de calorías que no corresponde con la composición nutricional del alimento, como el etiquetado de una zanahoria fresca que informa $310 \mathrm{cal} / 100 \mathrm{~g}$.

En el caso del etiquetado voluntario de la composición nutricional, $81,7 \%$ de la información es incorrecta.

\section{Información presumiblemente correcta}

Hemos estudiado la calidad de la aplicación del etiquetado nutricional al que obliga el Art 115 del RSA vigente de nuestro país, donde se debe informar el contenido de nutrientes en los productos que se comercializan envasados. Se encontró que $90,4 \%$ de la información nutricional obligatoria de los productos que se comercializan en Santiago es presumiblemente correcta.

Es importante señalar que, si bien la información entregada en la etiqueta de un producto es posiblemente correcta, no significa que sea exacta, ya que puede haber sido obtenida de una Tabla de referencia y no por análisis químico. La composición de vegetales y frutas cambia según un sin número de factores, como la variedad, el clima, $y$ el suelo de una región geográfica. Por otro lado, la composición de la carne y la leche varía según la raza y el tipo de alimentación de los animales, entre otros. Lo mismo ocurre con la composición de los pescados. Pese a esto, la información obtenida mediante este tipo de Tablas de referencia permite tener una estimación del contenido de 
nutrientes del producto y ayuda al consumidor a hacer una elección más informada de los productos alimenticios.

Aparentemente, la mayoría de las grandes empresas de alimentos lácteos, cárneos, cecinas, cereales y productos derivados, y golosinas han optado por informar la composición química de sus productos mediante el análisis químico de éstos, sin recurrir a Tablas de referencia, puesto que no hay coincidencia exacta con ninguna de las Tablas utilizadas en este trabajo.

Solamente $9,6 \%$ de los productos estudiados presentó una información que se consideró incorrecta. El 42,4\% de los errores se debe a que en el reporte de hidratos de carbono disponibles, en realidad se informa el contenido de hidratos de carbono totales. Esta discrepancia se dedujo al comparar los datos de la etiqueta con la información de las Tablas de referencia desde donde inferimos habían sido copiados en forma directa, ya que los números coincidían exactamente. Este error, en su mayoría en vegetales y frutas, es fácilmente superable por los productores de estos alimentos, puesto que la información la obtienen de Tablas de referencia donde también aparece el contenido de fibra de dichos productos. El descuento del contenido de fibra de los carbohidratos totales permite obtener fácilmente el valor de los hidratos de carbono disponibles.

Parte de la información dudosa sugiere una falta de prolijidad en la transcripción de la información desde el documento que informa la composición del producto (ya sea el análisis químico del mismo o simplemente la Tabla de referencia) a la etiqueta del producto.

Los alimentos envasados vendidos por los supermercados -los cuales no están obligados a informar la composición nutricional- presentan $82 \%$ de inexactitud, aunque la Ley exige que si entregan información nutricional deben cumplir con el Reglamento correspondiente. En este sentido, es importante señalar que estas incorrecciones confunden al consumidor, siendo preferible no informar para evitar la entrega de una información errada.

En la información referente a los tipos de grasa de los alimentos se constató ambigüedad. En la mayoría de los productos las grasas saturadas, las mono insaturadas y las poli insaturadas dan cuenta de la grasa total del alimento, sin incluir en la suma a las grasas trans. Sin embargo, otro grupo de productos incluye a las grasas trans en la suma de la grasa total. En Estados Unidos de Norteamérica, la exigencia es informar sólo las grasas saturadas y las trans, lo que resulta más fácil de entender para el consumidor común ${ }^{3}$. Sin embargo, ya que en Chile se ha hecho el esfuerzo de entregar más información, sería importante definir exactamente el modo de informar los distintos tipos de grasas.

Otro elemento que confunde al consumidor es el uso de los nombres de los distintos ácidos grasos. En los ácidos grasos poli insaturados se usa indistintamente ácido linoleico y omega-6, lo mismo ocurre con el ácido alfa-linolénico, EPA, DHA y omega-3.

El gran esfuerzo que han hecho los productores de alimentos envasados en nuestro país, como consecuencia de la modificación del Art. 115 del RSA impulsado por el Ministerio de Salud, es un gran avance para Chile. Desde su entrada en vigor a fines del año 2006 se han realizado varias modificaciones, aquellas que se desprenden de la Ley 20.606 en las que se establecen los límites de los componentes críticos (sal, azúcar, grasas) para definir cuándo un producto es 'Alto en', y también la señalética informativa que deberá usarse en los envases, que aún no entra en vigor. Los avances científicos obligan a actualizar las normas con el fin de informar correctamente al consumidor, lo que requiere el esfuerzo conjunto y concertado del gobierno, la industria y los consumidores.

\section{Conclusiones}

1. Chile ha dado un paso importante con la reforma del Reglamento Sanitario de los Alimentos, Decreto 57/05 del Ministerio de Salud, poniéndose a nivel de los países de América del Norte y de la Comunidad Económica Europea.

2. Este gran esfuerzo que ha realizado Chile, principalmente por parte de la industria de alimentos, debe implementarse de modo que sea una herramienta eficaz para la selección de alimentos por parte del consumidor.

3. En general, los alimentos envasados por sus productores, que tienen la obligación de informar, cumplen correctamente con el etiquetado nutricional.

4. La mayoría de los productores de vegetales y frutas, y en general, los productos de empresas pequeñas y medianas han optado por poner la 
información proveniente de una Tabla certificada. La más utilizada es la Tabla de la USDA.

5. Es posible corregir gran parte de la información errada si las empresas que utilizan Tablas de referencia se asesoran en cómo utilizarlas para obtener la información que exige el RSA.

6. La industria de alimentos procesados debe enfocarse en el desarrollo de alimentos con bajos niveles de nutrientes críticos, invirtiendo en investigación con el fin de aumentar la oferta de alimentos saludables.

7. Es necesario educar al consumidor, no sólo en cómo leer la información nutricional, sino también en qué comer con mayor y menor frecuencia, para tener una alimentación equilibrada. Esta es una tarea difícil que requiere de la implementación de campañas publicitarias que involucran al gobierno, a la industria y a la academia.

\section{Referencias}

1. Kessler DA. The evolution of national nutrition policy. Annu Rev Nutr 1995; 15: 13-26.

2. SchneemanB, Trumbo P, Ellwood K, Satchell F. The regulatory process to revise nutrient labeling relative to the Dietary Reference Intakes. Am J Clin Nutr 2006; 83: 1228S-30S.

3. Office of Nutritional Products, Labeling, and Dietary Supplements in the Center for Food Safety and Applied Nutrition at the U.S. Food and Drug Administration. Guidance for Industry: A Food Labeling Guide. 2009. http://www.fda.gov/Food/GuidanceRegulation/GuidanceDocumentsRegulatoryInformation/LabelingNutrition/ucm2006828.htm

4. Borra S. Consumer perspectives on food labels. Am J Clin Nutr2006; 83: 1235S.

5. Fulgoni VL, Miller GD. Dietary Reference Intakes for food labeling. Am J Clin Nutr 2006; 83: 1215S-16S.

6. Murphy SP, Barr SI. Recommended Dietary Allowances should be used to set Daily Values for nutrition labeling. Am J Clin Nutr 2006; 83: 1223S-27S.

7. Tarasuk V. Use of population-weighted Estimated Average Requirements as a basis for Daily Values on food labels. Am J Clin Nutr 2006; 83: 1217S-22S.

8. Murphy SP, Barr SI Food guides reflect similarities and differences in dietary guidance in three countries (Japan, Canada, and the United States). Nutr Rev 2007; 65: 14148.

9. Asp NG, Bryngelsson S. Health claims in Europe: new legislation and PASSCLAIM for substantiation. J Nutr 2008; 138: 1210S-15S.

10. European Parliament 2006. Position of the European Parliament adopted at second reading on 16 may 2006 with a view to the adoption of Regulation (EC) $\mathrm{N}_{-}$ .../2006 of the European Parliament and of the Council on nutrition and health claims made on foods.

11. Quinio C, Biltoft-Jensen A, De Henauw S, Gibney MJ, Huybrechts I, McCarthy SN, et al. Comparison of different nutrient profiling schemes to a new reference method using dietary surveys. Eur J Nutr 2007; 46 Suppl 2: 37-46.

12. Volatier JL, Biltoft-Jensen A, De Henauw S, Gibney MJ, Huybrechts I, McCarthy SN, et al. A new reference method for the validation of the nutrient profiling schemes using dietary surveys. Eur J Nutr 2007; 46 Suppl 2: 29-36.

13. Ministerio de Salud, República de Chile. Encuesta Nacional de Salud ENS Chile 2009-2010 http://web. minsal.cl/portal/url/item/bcb03d7bc28b64dfe04 0010165012d23.pdf

14. Ministerio de Salud, República de Chile. Reglamento Sanitario de los alimentos http://web.minsal.cl/portal/ url/item/d61a26b0e9043de4e0400101650149c0.pdf

15. Ministerio de Salud, Republica de Chile, Informe final estudio de carga de enfermedad y carga atribuible. http://epi.minsal.cl/epi/html/invest/cargaenf2008/Informe\%20final\%20carga_Enf_2007.pdf

16. Agricultural Research Service, United States Department of Agriculture. What's In The Foods You Eat Search Tool, 2013. http://www.ars.usda.gov/Services/docs. htm?docid $=17032$.

17. Mataix J, Mañas M (eds) Tabla de Composición de Alimentos Españoles. Granada. $3^{\circ}$ ed. Ed. Universidad de Granada. Granada 1998.

18. Schmidt-Hebbel H, Pennacchiotti I, Masson L, Mella MA. Tabla de Composición Química de Alimentos Chilenos. $8^{\circ}$ Ed. Facultad de Ciencias Químicas y Farmacéuticas. Universidad de Chile. Santiago, 1992. p781-86.

19. Chilealimentos 2011, http://www.chilealimentos.com/ link.cgi/Asociacion/Noticias_Chilealimentos/2011/ 17924. 